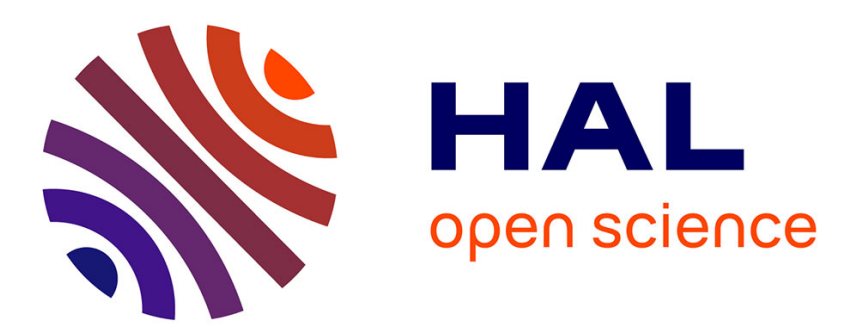

\title{
Les rythmes de la morphogénèse : leurs conceptions à travers l'histoire de la géomorphologie
}

\author{
Jean-Louis Ballais
}

\section{To cite this version:}

Jean-Louis Ballais. Les rythmes de la morphogénèse: leurs conceptions à travers l'histoire de la géomorphologie. Espace Géographique, 2000, 29 (2), pp.97-104. 10.3406/spgeo.2000.1980 . hal01569081

\section{HAL Id: hal-01569081 \\ https://hal-amu.archives-ouvertes.fr/hal-01569081}

Submitted on 26 Jul 2017

HAL is a multi-disciplinary open access archive for the deposit and dissemination of scientific research documents, whether they are published or not. The documents may come from teaching and research institutions in France or abroad, or from public or private research centers.
L'archive ouverte pluridisciplinaire $\mathbf{H A L}$, est destinée au dépôt et à la diffusion de documents scientifiques de niveau recherche, publiés ou non, émanant des établissements d'enseignement et de recherche français ou étrangers, des laboratoires publics ou privés. 


\title{
Jean-Louis Ballais
}

\section{Les rythmes de la morphogénèse : leurs conceptions à travers l'histoire de la géomorphologie}

In: Espace géographique. Tome 29 n², 2000. pp. 97-104.

\begin{abstract}
The pace of morphogenesis: its conception through the history of geomorphology. - The pace of morphogenesis has been a central issue in geomorphology since the discipline became a science in its own right. The major paradigms of catastrophism and uniformitarianism appeared before Davis. In Davisian theory, the evolution of landforms begins with a catastrophic event, but a continuous process is the main concept. After Davis, dynamic geomorphology and the concept of systems of denudation (and morphogenesis) became predominant, before being subsequently enhanced by neo-catastrophism.
\end{abstract}

Résumé

Le problème des rythmes de la morphogénèse est au cœur de la réflexion épistémologique depuis la constitution de la géomorphologie en science autonome. Avant Davis, les grands paradigmes du catastrophisme et de l'actualisme émergent. Avec Davis, si l'évolution du relief commence par une catastrophe, c'est ensuite la continuité de l'érosion qui domine. Après Davis, la géomorphologie dynamique et le concept de système d'érosion (puis morphogénique) s'imposent puis s'enrichissent des apports d'un néocatastrophisme.

Citer ce document / Cite this document :

Ballais Jean-Louis. Les rythmes de la morphogénèse : leurs conceptions à travers l'histoire de la géomorphologie. In: Espace géographique. Tome $29 \mathrm{n}^{\circ} 2$, 2000. pp. 97-104.

doi : $10.3406 /$ spgeo.2000.1980

http://www.persee.fr/web/revues/home/prescript/article/spgeo_0046-2497_2000_num_29_2_1980 


\title{
Les rythmes de la morphogénèse : leurs conceptions à travers l'histoire de la géomorphologie
}

\author{
Jean-Lovis Ballais \\ Institut de Géographie, UMR ESPACE 5651, UFR Sciences géographiques et aménagement, \\ 29 avenue Robert Schuman, 13621 Aix-en-Provence cedex 1
}

RÉSUMÉ- - Le problème des rythmes de la morphogénèse est au cœur de la réflexion épistémologique depuis la constitution de la géomorphologie en science autonome. Avant Davis, les grands paradigmes du catastrophisme et de l'actualisme émergent. Avec Davis, si l'évolution du relief commence par une catastrophe, c'est ensuite la continuité de l'érosion qui domine. Après Davis, la géomorphologie dynamique et le concept de système d'érosion (puis morphogénique) s'imposent puis s'enrichissent des apports d'un néocatastrophisme.

ACTUALISME, CATASTROPHISME, ÉROSION, NÉOCATASTROPHISME
ABSTRACT. - The pace of morphogenesis: its conception through the history of geomorphology. - The pace of morphogenesis has been a central issue in geomorphology since the discipline became a science in its own right. The major paradigms of catastrophism and uniformitarianism appeared before Davis. In Davisian theory, the evolution of landforms begins with a catastrophic event, but a continuous process is the main concept. After Davis, dynamic geomorphology and the concept of systems of denudation (and morphogenesis) became predominant, before being subsequently enhanced by neo-catastrophism.

CATASTROPHISM, EROSION, NEO-CATASTROPHISM, UNIFORMITARIANISM

\section{Introduction}

La réflexion épistémologique française sur la géomorphologie a connu un temps fort dans les années 1950-1970 pendant lesquelles s'effectue et s'approfondit définitivement la coupure épistémologique entre la géomorphologie davisienne et la géomorphologie dynamique-climatique. Cette période est marquée par la publication des seuls ouvrages consacrés aux questions de théories et de méthodes : dans l'ordre chronologique, les ouvrages célèbres de P. Birot (1955) Les Méthodes de la morphologie et de J. Tricart
(1965) Principes et méthodes de la géomorphologie, suivis du petit volume injustement négligé et au titre provocateur, publié par A. Reynaud (1971), Épistémologie de la géomorphologie. C'est aussi pendant cette période que $\mathrm{H}$. Baulig publie ses Essais de géomorphologie (1950) et A. Cholley ses Recherches morphologiques (1957).

Depuis, les réflexions épistémologiques sont devenues très rares, se limitant à de courts articles, à l'exception toute récente des Composantes et concepts de la géographie physique sous la direction de M. Derruau (1996). Faut-il 
penser que ce long silence à peu près total correspond au triomphe de la géomorphologie dynamique et climatique sur la géomorphologie davisienne et que, par conséquent, la discussion devenait inutile? La réalité est probablement plus complexe comme le montre la poursuite de la réflexion engagée par $\mathrm{C}$. Klein $\mathrm{Du}$ dynamisme des processus à la dynamique des formes (1993).

Le but poursuivi dans ce texte n'est pas de tenter d'approfondir la réflexion épistémologique sur l'ensemble de la géomorphologie en tant que science, ce qui devrait relever d'un travail collectif de grande ampleur. Il s'agit d'apporter des éléments de réflexion sur la conception des rythmes de l'érosion, des rythmes des processus morphogéniques, telle qu'elle est apparue dans les théories successives en géomorphologie. C'est dire que, même si cette réflexion s'appuie préférentiellement sur des exemples français, elle ne saurait en aucune manière représenter la vision d'une école française de géomorphologie. L'importance historique de la théorie de W.M. Davis impose d'en faire le pivot central de toute réflexion épistémologique. On peut ainsi distinguer la période avant Davis, la théorie de Davis et la période après Davis. L'exposé des théories successives obligera à utiliser le vocabulaire propre de ces théories afin d'en expliciter le contenu; cela ne signifie évidemment pas que j'en reprends à mon compte les concepts et le vocabulaire.

\section{La période avant Davis}

Pour le détail de la période précédant Davis, je renvoie aux ouvrages de R.J. Chorley et al. (1964), J. Tricart (1965), A. Reynaud (1971) et au texte de N. Broc dans M. Derruau (1996), texte qui néglige cependant l'apport de la civilisation musulmane médiévale en général, et celui d'Ibn Sina (Avicenne) en particulier.

\section{Du catastrophisme à l'actualisme}

J. Tricart (1965), en particulier, montre la naissance de la géologie moderne en Angleterre au XVII ${ }^{\mathrm{e}}$ siècle, pour résoudre certains problèmes d'exploitation du charbon, par exemple l'existence de discordances. Se pose alors le problème des processus responsables de la troncature des strates et en conséquence les préoccupations de géodynamique externe ou de géomorphologie commencent à émerger. Une explication possible est alors recherchée dans les références bibliques, sous la forme du Déluge, c'est-à-dire, de mon point de vue, une courte période d'extrême efficacité des processus morphogéniques qui auraient ainsi aplani des reliefs en quelques dizaines de jours. Le catastrophisme en géomorphologie était né.

En opposition à cette vision catastrophiste appuyée sur l'autorité sacrée de la Bible, Hutton puis Playfair, à la fin du XVIII ${ }^{e}$ siècle, avancent les premiers éléments de l'actualisme déjà esquissés dans des travaux de la première moitié de ce siècle : le relief nivelé par la surface de discordance a été arasé par les actions (processus) actuellement observables à la surface du globe. Plus tard et en France, A. Surell (1841) étudie les processus torrentiels actuels, premier grand exemple de géomorphologie dynamique, et montre leur efficacité en l'absence de forêt.

\section{L'uimpossible » actualisme}

Les idées éparses précédentes sont regroupées et systématisées par C. Lyell à partir de 1830 sous le nom d'actualisme (appelé aussi uniformitarisme depuis 1932) ou théorie des causes actuelles : le relief évolue depuis toujours sous l'action de forces érosives du même type que celles qui agissent sous nos yeux et qu'il est facile d'étudier. Dans le détail, et sans que l'articulation entre climat et processus soit toujours explicitée, l'actualisme s'applique aussi au climat considéré comme un facteur uniforme et stable, donc neutre.

L'actualisme présente un avantage considérable, sur le plan théorique : il évite les excès de l'imagination et oblige, en principe, à une étude très fine de la réalité. Par ailleurs, il théorise l'idée que le relief se transforme, que les paysages changent. Il rompt ainsi avec le «réalisme naif » (A. Reynaud), mais les deux théories s'accordent pour ne pas donner d'importance aux phénomènes catastrophiques. Pour le réalisme, les éboulements, les avalanches, les glissements de terrain, les ravinements, les inondations ne concernent que certaines régions et ne modifient pas, en général, des paysages globalement immuables. Pour l'actualisme, ces mêmes phénomènes catastrophiques ne sont, malgré leur magnitude, que des épiphénomènes qui ne sont pas représentatifs des forces érosives dominantes. Ainsi, d'un certain point de vue, l'actualisme rejette doublement le catastrophisme : il rejette le Déluge comme phénomène morphogénique majeur dans 
sa durée très brève, et il rejette les phénomènes catastrophiques dans la durée plus longue. On peut donc considérer qu'il prépare le terrain à une vision davisienne très éloignée de l'observation des processus autres que fluviatiles.

L'actualisme s'est imposé car comment connaître le passé si le présent ne l'est pas? Mais, d'un autre côté, l'actualisme présente un défaut théorique majeur : il n'est vrai que dans la mesure où les phénomènes érosifs actuels sont les mêmes que ceux du passé et présentent les mêmes caractéristiques et la même intensité. «Dans le cas contraire, [l'actualisme] revient à fausser l'explication des formes du relief et à commettre des erreurs de raisonnement aussi graves que celles du réalisme.» (A. Reynaud, 1971, p. 25).

\section{La théorie de Davis}

La théorie de Davis, théorie du cycle d'érosion, a fait l'objet d'abondantes publications et analyses; tout particulièrement, R.J. Chorley et al. (1973) lui ont consacré le deuxième volume de The History of The Study of Landforms. Comme pour les théories précédentes, je ne m'y intéresserai que du point de vue des rythmes de l'érosion. Il est très clair que le cycle d'érosion commence par un événement catastrophique, la brusque déformation d'une surface plane par une action tectonique (G. Viers, 1967, p. 111). On reconnaît là l'influence d'un catastrophisme tectonique hérité de la Bible et qui avait cours à la fin du XVIII ${ }^{\mathrm{e}}$ siècle chez E. Kant et au début du XIX ${ }^{\mathrm{e}}$ siècle chez de nombreux géologues, tel G. Cuvier. Ensuite, après cet événement catastrophique, et tout au contraire, le cycle se déroule sous l'influence d'une action de l'érosion lente, prolongée, continue, qui exclut tout catastrophisme. Cette évolution peut être interrompue ou relancée par un nouveau mouvement tectonique, toujours brusque et donc de courte durée, qui perturbe, de l'extérieur, l'évolution normale du cycle d'érosion. Les accidents interrompent l'harmonie d'une évolution continue, l'empêchent d'arriver à son terme, mais en même temps la relancent. Ces catastrophes ont, de plus, le mérite de justifier les discontinuités très évidentes et de les réduire aux conséquences d'un cataclysme, donc d'en faire des événements exceptionnels qui ne présentent que peu d'intérêt. «La discontinuité est subie de l'extérieur comme quelque chose d'inévitable mais de gênant, de surajouté, d'imprévu, quelque chose qui déchire le cours du temps et qui surprend par son caractère irrationnel. Elle ne fait pas partie intégrante d'une évolution qu'elle bouleverse en la troublant. Elle n'est acceptée que pour mieux mettre en valeur la prédominance absolue de la continuité dont elle est l'antithèse nécessaire» (A. Reynaud, 1971, p. 76-77).

Cette continuité permet, pour la première fois de façon relativement fondée, de prévoir l'avenir (et d'en tirer des conclusions apocalyptiques). C'est ainsi que, selon $\mathrm{C}$. Vélain dans La Grande Encyclopédie (1885-1902) (in A. Reynaud, 1971), le Mississippi rejette chaque année 28 millions de tonnes de limons dans le golfe du Mexique, d'où il en conclut que le relief d'Amérique du Nord aurait disparu dans 4,5 millions d'années. «Avec le Gange qui travaille plus vite, il suffirait de 930000 années pour raser l'Asie [...]. Si l'activité des agents extérieurs demeurait toujours ce qu'elle est aujourd'hui, 4,5 millions d'années suffiraient pour raboter (sic) complètement la surface de la terre.» (idem).

\section{Après Davis}

\section{Système d'érosion et géomorphologie dynamique}

Avec la charnière du milieu du $\mathrm{XX}^{\mathrm{e}}$ siècle, apparaissent de nouveaux concepts qui constituent la base théorique de la contestation du modèle davisien, remis en cause, dans les faits, par les recherches de A. Strahler et S.A. Schumm $(1954,1956)$, et de la formulation d'un nouveau paradigme : système d'érosion (puis système morphogénique), échelle, seuil. Le concept-clé de système d'érosion apparaît sous la plume d'A. Cholley (1950) : «Comme l'ont montré tout à l'heure l'exemple de l'érosion glaciaire et celui de l'érosion désertique, c'est tout un complexe d'agents, c'est un véritable système d'érosion que chaque climat met en œuvre»; «L'action des facteurs d'érosion varie suivant les conditions du climat; ce n'est pas en les considérant isolément que nous parvenons à saisir convenablement la réalité, mais en recherchant comment ils s'enchaînent et en retrouvant le lien qui les unit dans l'ensemble morphologique qui résulte de leur intervention.» À la vision d'une érosion manquant de substance et agissant avec régularité et constance, se substitue ainsi un système de processus morphogéniques dont chaque élément ne fonctionne pas obligatoirement de manière continue, de telle sorte que l'efficacité du système, 
résultante de celle de ses éléments constitutifs, varie au cours du temps : au continu se substitue le discontinu. Les discontinuités résultent du passage de seuils dont la typologie a été proposée plus tard par R. Brunet (1968). Ainsi, les phénomènes, les processus de forte magnitude, sont-ils réintroduits dans la géomorphologie dynamique et peuvent même devenir objet d'études spécifiques. «L'attention doit se porter sur les phénomènes extrêmes : chaque alternance gel-dégel, chaque crue, chaque averse n'ont pas automatiquement des conséquences morphogéniques.» (A. Reynaud, 1971, p. 49).

J. Tricart (1965, p. 108-110), très tôt, théorise cette nécessité de profiter des phénomènes extraordinaires pour en faire une étude détaillée, nécessité qu'il met en pratique concernant les effets de la crue de 1957 dans le Queyras : « Il se produit, de temps à autre, des phénomènes qui, dans l'ordre de grandeur des durées où nous nous plaçons, sont hors série. Ils ont très peu de chance de se reproduire, car ils résultent d'un concours de circonstances exceptionnel, généralement imprévisible. Mais ils n'en revêtent pas moins une très grande importance géomorphologique. Ils créent souvent des modifications durables, qui ne s'effacent que lentement et qui se traduisent par un déséquilibre persistant, une altération des mécanismes morphogénétiques qui régnaient auparavant.»Il est donc nécessaire de les analyser soigneusement et de les replacer aussi exactement que possible dans leur contexte morphogénique, en particulier dans la perspective de définir les moyens d'un nouvel aménagement. Une difficulté majeure est de bien situer de tels phénomènes dans le système morphogénique, de ni les sous-estimer (ce qui était le cas général), ni les surestimer (ce qui deviendra un objet de débat plus tard). J. Tricart insiste tout spécialement sur la nécessité de déterminer les «concours de circonstances» déclencheurs et les «phénomènes de résonance» amplificateurs. Il préconise aussi un suivi en détail de l'évolution du «traumatisme». Il s'oppose ainsi aux vues de L.B. Leopold et al. (1964) qui privilégient les magnitudes moyennes dans la morphogénèse fluviale.

En distinguant deux types de coupures, à propos de l'histoire, A. Reynaud (1971) oppose les coupures réelles, celles qui se traduisent par des réorganisations plus ou moins totales, mais qui, souvent peu apparentes, sont donc fréquemment ignorées ou oubliées, et les coupures accidentelles. Ces dernières, instantanées, brutales, ne remettent rien en cause car elles sont d'origine extérieure (c'est-à-dire qu'elles résultent des aléas de la conjoncture, comme les événements militaires ou les crises économiques). Ces coupures qui, au contraire des précédentes, sont vivement ressenties, ne sont pourtant pas fondamentales : «crise tectonique ou crise économique, avalanche ou épidémie ont des caractères communs ». En me limitant aux systèmes morphogéniques, je ferai deux remarques vis-à-vis de cette affirmation très structuraliste :

- la première est que rien ne permet, a priori, d'exclure une avalanche d'un système morphogénique montagnard. Au contraire, elle peut constituer un des processus et même un des processus majeurs. Son importance est alors appréciée en termes de bilan d'ablation et d'accumulation par rapport aux autres processus, pendant une période donnée;

- la deuxième est que la magnitude exceptionnelle d'un processus peut faire «basculer» l'ensemble du système morphogénique, soit provisoirement (à une échelle temporelle variable, par exemple de quelques heures à quelques années), soit définitivement (à l'échelle humaine ou séculaire). En fait, à ce propos, se pose ici le problème de la reconnaissance des éléments constitutifs du système morphogénique dont une bonne illustration est donnée par les incendies de forêt, tout particulièrement dans le domaine méditerranéen (Ballais, Bosc, 1994).

Cependant, la réalité des recherches géomorphologiques françaises, si elle a très majoritairement accepté le concept de système d'érosion (puis de système morphogénique), a très peu retenu le rôle des phénomènes catastrophiques dans l'efficacité de ces systèmes. Restes de la théorie davisienne? Méconnaissance des processus actuels et de leur efficacité alors même que se développait aux États-Unis puis en Grande-Bretagne le courant de la «géomorphologie des processus» ou géomorphologie dynamique, devenu maintenant dominant au niveau mondial? Ou tout cela et d'autres raisons plus générales?

\section{Le néocatastrophisme}

C'est sous l'influence d'un néocatastrophisme (Ballais, 1983) que l'intérêt des géomorphologucs français va sc porter à nouveau sur les processus de forte magnitude. $\mathrm{Ce}$ néocatastrophisme se veut scientifique, il n'a plus rien à voir avec celui d'origine biblique qui n'est plus défendu dans les pays développés que par quelques sectaires. Il 
apparaît en France, au sein des sciences de la terre, au début des années 1980 où il sous-tend l'organisation de colloques successifs sur la composante "endodynamique» dans la morphogénèse, les événements exceptionnels et leur enregistrement dans les séries sédimentaires, la paléoclimatologie et les crises du milieu naturel, les effets des séismes sur les reliefs de forte énergie, les mouvements de terrain catastrophiques, ou encore des numéros de revue tel le $\mathrm{n}^{\circ} 24$ de Hérodote (1982) «Terres à hauts risques». Bien évidemment, ces différentes manifestations ne font pas référence aux catastrophes de la même manière et il y aurait lieu d'apporter bien des nuances, tout particulièrement entre celles qui se sont intéressées à la magnitude des phénomènes et celles qui ont insisté sur l'impact sur les populations humaines et sur leurs biens, c'est-à-dire plus sur le concept de risque naturel qui émerge puissamment à ce moment. De plus, les titres successifs de certains colloques montrent bien les hésitations face aux concepts de catastrophe et de crise : c'est ainsi que le colloque sur la paléoclimatologie et les crises du milieu naturel s'est tenu finalement sous le titre "Anomalies et crises climatiques" et celui sur les mouvements de terrain catastrophiques est devenu simplement «Mouvements de terrain».

Même si le hasard existe, bien sûr, ce n'est cependant pas tout à fait par hasard si ce néocatastrophisme s'est développé quelques années après le début de la plus grande crise économique du système capitaliste depuis 1929. D'autant que cette crise est survenue après les «trente glorieuses", une période exceptionnellement longue de croissance économique, parfois irrégulière, depuis la seconde guerre mondiale, période qui avait favorisé la réflexion sur la longue durée dans le sillage de Braudel (1966) et, selon toute vraisemblance, sur le structuralisme.

Cependant, cette période n'était pas exempte de contradictions, en particulier dans l'évolution des théories de la géomorphologie, pourtant l'un des premiers domaines où le structuralisme a été appliqué (Cholley, 1950), avant même qu'il ait été théorisé par C. Lévi-Strauss ou M. Foucault en particulier. Cette période, nous venons de le voir, se caractérise en effet par la mise à bas de la prééminence de la théorie davisienne, non pas tant en raison du cataclysme initial qu'elle suppose, qu'à partir des ruptures, des discontinuités, des accélérations repérées dans la formation du relief et du modelé par des recherches plus méthodiques ou/et à partir de présupposés théoriques différents qui montrent la fausseté de la continuité du cycle d'érosion et de la décélération régulière qu'il suppose.

La «crise» économique qui est apparue de manière brutale en 1973, révélée par le «choc» pétrolier, a provoqué un profond ébranlement social et idéologique dont les effets ne sont pas encore tous épuisés aujourd'hui. C'est d'abord dans les domaines les plus sensibles des médias et de la culture (littérature populaire, radio, cinéma) que la «crise» a produit ses premiers effets : multiplication des ouvrages et des émissions sur la sorcellerie, le paranormal, les énigmes naturelles ou historiques, et surtout l'apparition et le développement extraordinaire des films catastrophes. Ces films mettent en cause d'abord et surtout la responsabilité des sociétés humaines, mais aussi celle des facteurs naturels (raz de marée $=$ tsunami, tremblements de terre $=$ séismes, etc. $)$.

Les sciences de la terre, avec un certain retard, ont suivi cc mouvement profond. Alors qu'elles pratiquent généralement un actualisme mesuré, chacune dans leur domaine (par exemple en sédimentologie ou en géomorphologie dynamique), elles tendent alors à se polariser sur l'étude des crises naturelles, pratiquant ainsi, sans le savoir et à une autre échelle, une forme d'actualisme étroit par rapport au contexte économique. Au niveau mondial, la formidable controverse sur les causes de l'extinction des dinosaures à la fin du Crétacé en est l'exemple emblématique. En France, la promulgation de la loi Tazieff (13 juillet 1982) a orienté durablement toute une partie de l'activité des géomorphologues en direction de l'étude des risques naturels dus aux aléas de forte magnitude ou de vitesse importante.

Ce néocatastrophisme, à la différence de l'ancien, ne s'affirme pas, au début, comme théorie. Il résulte d'initiatives, de réflexions qui se posent comme spontanées et non coordonnées, voire totalement inconscientes d'une liaison avec la «crise» économique. Ou, pire, niant toute liaison avec cette «crise» économique comme si les chercheurs étaient de purs esprits perdus dans les nuées (ah! le mythe du savant Cosinus !) et comme si le «mouvement des idées», pour reprendre une expression traditionnelle, avait sa propre logique indépendante. Mais, l'idéologie produisant ses effets, de plus en plus de chercheurs avancent des éléments théoriques, tout spécialement dans les zones arides et dans le domaine méditerranéen. Par exemple, 
Cooke et al. (1993, p. 98) affirment que, dans les zones arides, ce sont les averses occasionnelles localement intenses qui peuvent être l'agent de changement le plus efficace, en contraste avec la zone tempérée où c'est l'événement de taille moyenne qui est souvent plus significatif. Tout récemment (Ballais, 1997), plusieurs chercheurs affirment le rôle décisif des événements de magnitude extrême dans le domaine méditerranéen, qu'il s'agisse du ruissellement en Espagne, de l'écoulement en Israël et au Maroc ou des mouvements de terrain au Maroc. R.J. Huggett est devenu l'un des observateurs les plus conséquents du catastrophisme, dans une série d'articles et d'ouvrages parus depuis 1988. En particulier (Huggett, 1994), il s'est interrogé sur l'apparition d'un néo-diluvianisme dont les bases scientifiques sont constituées par des formes ou des dépôts fossiles littoraux dus à d'énormes tsunamis ou à des supervagues, d'une part et, d'autre part, des formes et dépôts continentaux dus au passage de très grandes quantités d'eau soudainement libérées. Je me limiterai à ce deuxième cas qui regroupe plusieurs types de ruptures de barrages dans l'ouest des États-Unis et du Canada tel le Bonneville Flood, environ 15000 ans BP en liaison avec des langues glaciaires ou, selon Hunt, avec l'impact de comètes ou d'astéroïdes. Les travaux de Baker et de son équipe sur les superfloods holocènes ou historiques d'Inde ou d'Australie (région d'Alice Springs en particulier) se placent dans cette même perspective (Pickup, 1991).

Sur un plan plus paradigmatique, il est encore trop tôt pour pleinement caractériser les recherches inspirées par la théorie du chaos déterministe issue des travaux du climatologue Lorenz en ce qui concerne les processus (et la géométrie fractale en ce qui concerne les formes, Dauphiné, 1995), mais l'existence de systèmes non linéaires est de plus en plus admise, ainsi que leur sensibilité aux conditions initiales. Un effort de réflexion est en cours dans ce sens (K. Richards, sous presse), qui devrait pouvoir permettre de mieux intégrer et donc de mieux quantifier l'importance des héritages pour le processus qui agit sur eux.

Ce néocatastrophisme permet d'approfondir, cette fois avec l'appareil théorique, conceptuel et technique du dernier quart du $\mathrm{Xx}^{\mathrm{e}}$ siècle, un des concepts-clés des sciences en général, et des sciences de la terre en particulier, celui de discontinuité. De ce point de vue, il est prometteur car il a déjà permis, par exemple, de repréciser l'originalité fondamentale du Quaternaire, période de crise à l'échelle géologique, aussi bien par la vigueur de la tecto-orogénèse que par l'ampleur et le rythme des variations climatiques, portant un coup décisif, s'il en était encore besoin, à l'actualisme vulgaire. Sur les questions étroitement liées de découpage temporel et de disparition de faunes, la polémique lancée depuis 1980 par l'hypothèse de la disparition d'origine catastrophique des dinosaures à la fin du Secondaire-Crétacé, a produit une énorme somme de nouvelles données, très supérieure à ce qui aurait été produit en l'absence de l'aiguillon de cette théorie.

\section{La géomorphologie dynamique ef l'uimpossible " actualisme}

Le développement spectaculaire, et au niveau mondial, de la géomorphologie dynamique, de la géomorphologie des processus, tire son origine d'un faisceau de nécéssités :

- relicr la science ct la technique, la théorie et la pratique dont la séparation constituait pour A. Reynaud (1971) le «drame actuel de la géomorphologie»; ceci par le développement d'une géomorphologie appliquée à l'aménagement (parmi de nombreux exemples, Masson et al., 1995), - bien connaitre le présent pour bien interpréter le passé, - bien connaître le présent pour bien prévoir le futur, en particulier dans la perspective des changements globaux sous l'influence de l'effet de serre.

Et ainsi se pose avec encore plus d'acuité le problème de l'impossible actualisme, sous la forme du passage de l'étude des processus, connaissables, quantifiables, reproductibles au moins partiellement, aux surfaces de discordance ou aux surfaces d'aplanissement. Est-ce que la micromorphologie appuyée sur la micromorphométrie donnera la clef de la macromorphologie? En d'autres termes, peut-on étudier de la même façon le processus élémentaire et le relief d'une région? Il y a plus de 30 ans déjà, J. Tricart (1965, p. 61-62) soulignait que «Lyell s'est avéré incapable de faire le lien entre les actions contemporaines qu'il décrit et les durées géologiques et de voir dans les surfaces de discordance tronquant d'anciennes montagnes le résultat des phénomènes actuels». Il soulignait également que, malgré les apports considérables des travaux de Ramsay et, surtout, de G.K. Gilbert sur les processus actuels, la géomorphologie, dans le dernier quart du XIX ${ }^{\mathrm{e}}$ siècle, ne parvenait toujours pas à dominer les énormes différences 
d'échelle qui séparent les processus actuels et les aplanissements à l'échelle géologique, résultant d'évolutions durant des millions d'années. Mais après que deux ou trois générations de géomorphologues aient consacré des milliers de pages à la description des surfaces d'aplanissement, recherche à laquelle j'ai participé, la même insatisfaction demeure : la compréhension de la genèse de telles surfaces à partir de la connaissance des processus actuels se heurte toujours à l'obstacle insurmontable du changement d'échelle spatiale (passage des objets géomorphologiques de $8^{\mathrm{e}}$ à $5^{\mathrm{e}}$ grandeur modelés par les processus récents ou actuels, d'une part, à des objets de $4^{\mathrm{e}}$ grandeur, d'autre part) et du changement d'échelle temporelle (Lageat $e t$ al, 1996, p. 235-246). Cela rappelé, la vogue (vague) actuelle de géomorphologie quantitative permet de préciser le vocabulaire et de l'enrichir, de retrouver ou d'affiner les seuils critiques, de clarifier les discontinuités, de mettre en valeur les rapports, d'établir les corrélations. Au lieu de se laisser emporter par des mots qui n'ont pas exactement le même sens d'un auteur à l'autre, en particulier des mots qui ne respectent pas toujours la notion d'échelle, la précision chiffrée ordonne les idées, oriente les hypothèses en écartant celles qui sont incompatibles avec elle. La donnée quantitative permet souvent de dépasser la subjectivité personnelle. Elle met à bas les illusions entretenues par l'affirmation selon laquelle n'importe quel individu peut, par simple observation visuelle du terrain effectuée à partir de repères, comprendre les modifications locales d'un lit fluvial ou d'un versant (Reffay, 1996).

La recherche de la quantification a accéléré la tendance au développement de la géomorphologie appliquée, apparue en France après la seconde guerre mondiale. L'avantage de l'application est d'orienter la recherche et la démonstration. Prévoir le tracé d'une route, préserver un village contre les risques d'avalanche, lutter contre l'érosion des sols, les glissements de terrain ou la désertification, oblige à mettre l'accent sur les processus, à les reconnaitre, à les analyser, à les mesurer. La nécessité impérieuse de la précision (car les erreurs auraient des conséquences graves) permet ainsi une étude fine des formes à petite échelle et des équilibres dynamiques. L'inconvénient de l'application est le risque de verser, d'une manière ou d'une autre, dans un actualisme sans rivage, en d'autres termes d'accorder trop d'importance relative à des phénomènes mineurs mais spectaculaires car actuels et concernant les sociétés humaines.

\section{Le concept de crise morphogénique}

Le concept de crise morphogénique s'est imposé, au contraire, sans difficulté, en liaison avec celui de crise climatique. Lorsqu'une crise climatique se déclenche, à la suite d'un changement de climat, elle entraîne une transformation du système morphogénique qui produit souvent une intensification de la morphogénèse durant un certain temps (Riser, 1995; Reffay, 1996) car la rupture d'équilibre favorise généralement la mobilisation des débris produits ou accumulés précédemment. Puis un nouvel équilibre dynamique s'établit sans que les conditions morphogéniques changent, ce que G. Viers $(1967$, p. 55) appellait le «dépérissement des systèmes d'érosion».

Le concept de crise climatique a été trop utilisé, alors que ce qui est constaté, c'est une crise morphogénique. À la limite, on aboutirait à un simple renversement de la géomorphologie davisienne : dans un cas, des crises tectoniques brutales s'opposent à la régularité dans les processus et dans l'intensité de l'évolution morphogénique, dans l'autre cas, des crises climatiques s'opposent à des mouvements tectoniques étalés dans le temps, bien que non permanents. Dans les deux cas, l'évolution dépend de crises, c'est-à-dire qu'elle est en rapport avec une conception catastrophiste. Cependant, les deux conceptions ne sont pas assimilables : dans la conception davisienne, la crise tectonique ne fait pas intrinsèquement partie du cycle d'érosion, tandis que dans la conception post-davisienne, le changement climatique provoque un changement interne : c'est le système morphogénique qui est transformé. Pour A. Reynaud (1971), la notion de crise climatique a l'avantage de mettre l'accent sur la relative rapidité du passage d'un système morphogénique à un autre, mais il faut bien rappeler que ce passage peut résulter d'un très léger changement d'une variable climatique de part et d'autre d'un seuil critique (le déclenchement de la cryoclastie par exemple, en rapport avec une très légère diminution de la température; ou encore une faible variation de l'intensité des pluies). La crise morphogénique peut ainsi trouver son origine dans une variation climatique qu'il est difficile de qualifier de crise. Il faut alors bien indiquer la place de cette variation dans les fluctuations statistiques du système climatique : reste t-elle dans la variabilité de ce système ou y a t-il changement d'état du système? 


\section{Conclusion}

Le problème du rythme de la morphogénèse est au cœur de la pensée géomorphologique depuis que la géomorphologie émerge peu à peu à la fin du XVIII ${ }^{\mathrm{e}}$ siècle. Les théories successives mettent toujours en avant l'opposition entre des événements de forte magnitude et de courte durée (tectoorogénèse davisienne, processus morphogéniques) et des événements de faible magnitude et de longue durée (l'érosion normale davisienne, les systèmes morphogéniques modaux). Chaque théorie, malgré les ruptures épistémologiques qu'elle opère avec la ou les théories précédentes, en reprend des éléments, mais on n'observe pas de processus de dépassement, au sens où l'on peut dire qu'en physique la théorie de la relativité d'Einstein «dépasse» la théorie de la gravité de Newton. La géomorphologie reste encore une science jeune, mal assurée de ses bases théoriques. Le progrès de la réflexion épistémologique viendra probablement de deux orientations en plein essor. D'abord, la confrontation de la géomorphologie dynamique, déjà entamée, avec les problèmes d'application et d'aménagement du territoire avec, en particulier, l'approfondissement de la connaissance de l'actualisme et, ensuite, la confrontation avec l'histoire des sociétés humaines au sein d'une vaste géoarchéologie.

\section{Références}

Ballais J.-L. (1983). «Sur le néo-catastrophisme». Travaux $d u$ CREGEPE, 3, p. 3-5.

BALLAIS J.-L. éd. (1997). «Impact anthropique en milieu méditerranéen». Méditerranée, $\mathrm{n}^{\circ} 1.2,98 \mathrm{p}$.

Ballais J.-L., BosC M.-C. (1994). «The ignifracts of the SainteVictoire mountain (Lower Provence, France) », in M. SALA, J.-L. RuBIo eds, Soil erosion and degradation as a consequence of forest fires, p. 217-227.

BAuLiG H. (1950). Essais de géomorphologie. «Publications de la Faculté des Lettres de Strasbourg», fasc. 114.

BIROT P. (1955). Les Méthodes de la morphologie. Paris : PUF, $177 \mathrm{p}$.

BRAUDEL F. (1966). La Méditerranée et le monde méditerranéen à l'époque de Philippe II. Paris : A. Colin, $2^{\mathrm{e}}$ éd.

BROC N. (1996). « La géographie physique : aperçu historique», in M. DeRruau dir. Composantes et concepts de la géographie physique. Paris : A. Colin, p. 25-39.

BRUNET R. (1968). Les Phénomènes de discontinuité en géographie. Paris : CNRS, $117 \mathrm{p}$.
Cholley A. (1950). « Morphologie structurale et morphologie climatique». Annales de Géographie, ${ }^{\circ} 317$, p. 321-335.

Cholley A. (1957). Recherches morphologiques. Paris : A. Colin, $207 \mathrm{p}$.

ChoRley R.J., DunN A., Beckinsale R.P. (1964). The History of the Study of Landforms, vol. 1: Geomorphology before Davis. Londres : Methuen, $678 \mathrm{p}$.

ChoRley R.J., Beckinsale R.P., Dunn A.J. (1973). The History of the Study of Landforms, vol. II : The Life and Work of William Morris Davis. Londres : Methuen, 874 p.

COOKE R., WarRen A., Goudie A. (1993). Desert Geomorphology. Londres : UCL Press, $526 \mathrm{p}$.

DAUPHINÉ A. (1995). Chaos, fractales et dynamique en géographie. Montpellier : GIP RECLUS, $135 \mathrm{p}$.

DerRuAu M. dir. (1996). Composantes et concepts de la géographie physique. Paris : A. Colin, 256 p.

HugGETT R.J. (1994). «Fluvialism or diluvialism? Changing views on superfloods and landscape change». Progress in Physical Geography, 18 : 3, p. 335-342.

KıEIN C. (1993). Du dynamisme des processus à la dynamique des formes en géomorphologie. Gap : Ophrys, $188 \mathrm{p}$.

Lageat Y., André M.-F., Allée P. (1996). " Apports et limites de l'expérimentation", in M. Derruau dir. Composantes et concepts de la géographie physique. Paris : A. Colin, p. 235-246.

LEOPOLD L.B., Wolman M.G., Miller J.-P. (1964). Fluvial processes in geomorphology. San Francisco : W.H. Freeman and $\mathrm{Co}, 522 \mathrm{p}$.

Masson M., Garry G., Ballais J.-L. (1996). Cartographie des zones inondables. Approche hydrogéomorphologique. Paris : Les Éditions Villes et Territoires, $100 \mathrm{p}$.

PICKUP G. (1991). «Event Frequency and Landscape Stability on the Floodplain Systems of Arid Central Australia». Quaternary Science Reviews, 10 : 5, p. 463-473.

REFFAY A. (1996). « La géomorphologie : composantes et concepts épistémologiques», in M. Derruau dir. Composantes et concepts de la géographie physique. Paris : A. Colin, p. 57-69.

ReYNAUd A. (1971). Épistémologie de la géomorphologie. Paris : Masson, $127 \mathrm{p}$.

RISER J. (1995). Érosion et paysages naturels. Paris : Flammarion, $128 \mathrm{p}$.

SCнUмm S.A. (1954). Evolution of drainage systems in badlands at Perth Ambey. New York : Columbia Univ., VI-89 p.

Schumm S.A. (1956). «The role of creep and rainwash on the retreat of badland slopes». American Journal of Sciences, CCLIX, p. 693-706.

SuRell A. (1841). Étude sur les torrents des Hautes-Alpes. Paris.

TriCART J. (1965). Principes et méthodes de la géomorphologie, Paris : Masson, $496 \mathrm{p}$.

VIERS G. (1967). Éléments de géomorphologie. Paris : F. Nathan, 208 p. 